\title{
Comparison of Xpert MTB/RIF Assay and the Conventional Sputum Microscopy in Detecting Mycobacterium tuberculosis in Northern Thailand
}

\author{
Kanokwan Pinyopornpanish, ${ }^{1}$ Romanee Chaiwarith, ${ }^{1}$ Chansom Pantip, ${ }^{2}$ \\ Rassamee Keawvichit, ${ }^{2}$ Kanlaya Wongworapat, ${ }^{2}$ Phadungkiat Khamnoi, ${ }^{3}$ \\ Khuanchai Supparatpinyo, ${ }^{1,2}$ and Thira Sirisanthana ${ }^{2}$ \\ ${ }^{1}$ Department of Medicine, Faculty of Medicine, Chiang Mai University, Muang, Chiang Mai 50200, Thailand \\ ${ }^{2}$ Research Institute for Health Sciences, Faculty of Medicine, Chiang Mai University, Muang, \\ Chiang Mai 50200, Thailand \\ ${ }^{3}$ Central Diagnostic Laboratory, Chiang Mai University Hospital, Muang, Chiang Mai 50200, Thailand
}

Correspondence should be addressed to Romanee Chaiwarith; rchaiwar@gmail.com

Received 3 March 2015; Accepted 15 April 2015

Academic Editor: Paul R. Klatser

Copyright (C) 2015 Kanokwan Pinyopornpanish et al. This is an open access article distributed under the Creative Commons Attribution License, which permits unrestricted use, distribution, and reproduction in any medium, provided the original work is properly cited.

\begin{abstract}
Background. Despite low sensitivity in detection of Mycobacterium tuberculosis, sputum acid-fast smear remains the main diagnostic method. This study aimed to compare the diagnostic performance of Xpert MTB/RIF assay versus conventional sputum acid-fast smear. Materials and Methods. A cross-sectional study was conducted at Chiang Mai University Hospital, Thailand. Patients who were $\geq 15$ years old and had clinically suspected pulmonary tuberculosis were included. Results. 109 specimens from 57 patients were included. Using MGIT sputum culture as a reference standard, the sensitivity (SEN) and specificity (SPEC) for Xpert were 95.3\% (95\% CI, 84.2\%, 99.4\%) and 86.4\% (95\% CI, 75.7\%, 93.6\%). The SEN and SPEC for sputum acid-fast smear were 60.5\% (95\% CI, $44.4 \%, 75.0 \%)$ and $98.5 \%$ (95\% CI, 91.8\%, 100\%). Xpert had significantly higher sensitivity ( $p$ value $<0.001)$ and lower specificity ( $p$ value $=0.022$ ) than sputum acid-fast smear. Among 43 culture-proven $M$. tuberculosis specimens, sensitivity of Xpert was $100 \%$ $(95 \%$ CI, $86.7 \%, 100 \%)$ in acid-fast positive smears $(n=26)$ and $88.2 \%(95 \%$ CI, 63.5\%, 98.5\%) in acid-fast negative smears $(n=17)$. Conclusions. The good sensitivity and specificity of Xpert assay in detecting M. tuberculosis from sputum specimens may help in early diagnosis and treatment of pulmonary tuberculosis, particularly among patients who had acid-fast negative sputum smear.
\end{abstract}

\section{Introduction}

Tuberculosis (TB) is a major health problem across the world. Thailand is one of the high TB burden countries. In 2012, World Health Organization (WHO) estimated that the incidence of TB in Thailand was 119 cases per 100,000 populations with 9,200 annual deaths. Among all TB cases, 12,000 were infected with HIV, and there were 2,200 annual deaths [1]. Early diagnosis and prompt treatment of TB are crucial to reduce morbidity and mortality, secondary drug resistance, and transmission of TB.
Despite low sensitivity in detection of Mycobacterium tuberculosis, acid-fast sputum smear remains the main diagnostic method in most countries, especially in resourcelimited settings [2]. In HIV infected patients with pulmonary TB, $24-61 \%$ have acid-fast negative sputum smear [3]. Mycobacterial culture is the gold standard and the most sensitive method for TB diagnosis; however, the use in clinical practice is limited due to a slow turnaround time, biosafety requirements, and high cost $[4,5]$.

Recently, in 2011, WHO endorsed the wide use of Xpert MTB/RIF assay, a fully automated diagnostic molecular test 
using real-time polymerase chain reaction (PCR) technology to simultaneously detect $M$. tuberculosis and rifampicin resistance mutations in the rpoB gene. This assay can provide the results within 2 hours [6]. Several studies have demonstrated that Xpert assay is highly sensitive and specific in diagnosis of both pulmonary $[7,8]$ and extrapulmonary TB $[9,10]$. Furthermore, Xpert assay was shown to be cost-effective for TB diagnosis, compared to microscopy in low and middle income settings [11]. Therefore, Xpert assay is strongly recommended as the initial diagnostic test in individuals suspected of having multidrug resistant (MDR) TB and in those with $\mathrm{HIV} / \mathrm{TB}$ coinfection. It is also recommended as a follow-on test in TB-suspected patients with acid-fast negative sputum smear.

However, Xpert assay is not widely recognized as a diagnostic test for TB in Thailand. This study, therefore, was conducted to evaluate diagnostic performance of Xpert assay in northern Thai patients with clinically suspected pulmonary tuberculosis, using tuberculosis culture as a reference standard.

\section{Materials and Methods}

2.1. Study Design and Population. We conducted a crosssectional study at Chiang Mai University Hospital, Chiang Mai, Thailand, between September 2012 and November 2013. The inclusion criteria were male or female patients with: (1) age $\geq 15$ years; (2) clinically suspected pulmonary tuberculosis, which was defined as having 2 or more of the following symptoms: fever, chronic cough, weight loss, pleuritic chest pain, hemoptysis, and with or without abnormal chest radiograph compatible with pulmonary tuberculosis (cavitary lesion, infiltration, and miliary pattern); and (3) no history of receiving antituberculous drug within 3 months before enrollment.

2.2. Specimen Collection and Processing. The eligible patients were asked to provide at least 1 expectorated sputum specimen to the maximum of 3 specimens. Sputum acid-fast smear was performed on fresh specimen at the Central Diagnostic Laboratory, Chiang Mai University Hospital, Faculty of Medicine. The specimen was then decontaminated and separated into 2 samples which were blindly tested at 2 laboratory sites.

(a) Liquid-media mycobacterial culture of all sputum specimens using Mycobacteria Growth Indicator Tube (MGIT) method as a reference standard was performed at the Central Diagnostic Laboratory, Chiang Mai University Hospital.

(b) Xpert MTB/RIF assay (Cepheid) was performed at Mycobacterial Laboratory, Research Institute for Health Sciences (RIHES), Chiang Mai University. The method was performed according to manufacturer's instruction of Xpert MTB/RIF [12].

The specimen was excluded from the analysis if (1) it was an invalid sample for Xpert assay or sample error according to Cepheid package insert, or (2) the culture was contaminated.
2.3. Data Collection. Demographic data including age, sex, past medical history of lung diseases, and HIV serostatus were recorded. Clinical data including fever, days of fever, chest pain, dyspnea, hemoptysis, weight loss, cough, and the extrapulmonary sites of tuberculosis were collected. Laboratory results included sputum acid-fast smear, sputum mycobacterial culture results, sputum Xpert MTB/RIF results, and chest radiographic findings were collected. Sputum acid-fast smear results were categorized into positive (reported AFB found per field as $1+, 2+, 3+$, and $4+$ ) or negative. Sputum Xpert MTB/RIF results were also categorized into positive (reported Xpert detected as very low, low, medium, and high), or negative. Sputum cultures were reported as $M$. tuberculosis, NTM, contaminated culture, or no growth.

2.4. Statistical Analysis. Descriptive data were presented in percentages, mean $\pm \mathrm{SD}$, and median (IQR) as appropriate. The sensitivity, specificity, positive predictive value (PPV), and negative predictive value (NPV) of Xpert and sputum acid-fast smear were compared to tuberculosis culture as a reference standard using contingency $2 \times 2$ tables and exact binomial confidence intervals. The sensitivity and specificity between sputum smear and Xpert were compared using McNemar's test. Among culture-confirmed pulmonary tuberculosis, comparison of characteristics between patients with positive and negative sputum acid-fast smear was performed using $\chi^{2}$ test or Fisher's exact test for categorical data and Student's $t$-test or Mann-Whitney $U$ test for continuous data. Factors associated with negative sputum acid-fast smear were tested in univariate models. Factors with the $p$ value of $<0.10$ from univariate analysis were then tested in a multivariate logistic regression model.

All statistical analyses were performed using Stata statistical software version 10.0 (Stata Statistical Software: Release 10.0, Stata Corporation, College Station, TX, 2007).

This study was approved from the Faculty of Medicine, Chiang Mai University ethical committee.

\section{Results}

3.1. Demographic and Clinical Data. Sixty consecutive patients who met the inclusion criteria providing 127 sputum specimens were enrolled to the study. Of 127 specimens, 2 were invalid for Xpert, 2 were sample error, 14 were contaminated, and 11 grew NTM. After exclusion of 18 specimens, 109 sputum specimens from 57 patients were included in the analysis. Twenty-nine, 15, and 12 patients provided 1, 2, and 3 specimens, respectively.

Demographic data of 57 patients are shown in Table 1. Twenty-three patients (40.4\%) were female and the mean age was $55.6 \pm 20.1$ years. Thirteen patients $(22.8 \%)$ had underlying lung diseases and 15 patients (26.3\%) had HIV infection.

3.2. Identification of M. tuberculosis and Drug Susceptibility Test. Among 109 sputum specimens, M. tuberculosis was isolated by MGIT culture from 43 specimens (39.4\%). Xpert assay and sputum acid-fast smear yielded positive result in 
TABLE 1: Demographic data of 57 patients with clinically suspected pulmonary tuberculosis.

\begin{tabular}{lc}
\hline Demographic data & Patients $(n=57)$ \\
\hline Female & $23(40.4 \%)$ \\
Age (mean \pm SD) & $55.6 \pm 20.1$ \\
Underlying lung diseases & \\
$\quad$ Chronic obstructive pulmonary & $6(10.7 \%)$ \\
disease (COPD) & $4(7.1 \%)$ \\
Bronchiectasis & $3(5.4 \%)$ \\
Other chronic lung diseases & \\
HIV serostatus & $15(26.3 \%)$ \\
Positive & $40(71.9 \%)$ \\
Negative & $1(1.8 \%)$ \\
Not tested &
\end{tabular}

46 specimens (45.9\%) and 27 specimens (24.8\%), respectively. The median time to get the culture results was 45 days (IQR 42, 57 days), whereas the median time to get results from Xpert assay and sputum acid-fast smear was 6 days (IQR 3, 8 days) and 1 day, respectively.

From MGIT culture, 3 specimens from 1 patient showed INH resistance, 4 specimens from 2 patients showed streptomycin resistance, and 3 specimens from 1 patient showed both INH and rifampin resistance. Xpert assay failed to detect rifampin resistance in all 3 specimens with rifampin resistance.

\subsection{Performance of Xpert Assay and Sputum Acid-Fast Smear.}

Table 2(a) shows the two by two contingency table comparing between Xpert assay and sputum culture. Using sputum culture as a reference standard, the overall sensitivity, specificity, PPV, and NPV for Xpert assay were 95.3\%, 86.4\%, 82.0\%, and $96.6 \%$, respectively.

Table 2(b) shows the two by two contingency table comparing between sputum acid-fast smear and sputum culture. Using sputum culture as a reference standard, the overall sensitivity, specificity, PPV, and NPV for sputum acid-fast smear were $60.5 \%, 98.5 \%, 96.3 \%$, and $79.3 \%$, respectively.

The sensitivity, specificity, PPV, and NPV and their correspondent 95\% CIs are shown in Table 3. Xpert assay had statistically significant higher sensitivity than sputum acidfast smear ( $p$ value $<0.001$ ), whereas the specificity was statistically lower than sputum acid-fast smear ( $p$ value 0.022 ).

Among 43 culture-proven $M$. tuberculosis specimens, sensitivity of Xpert assay was 100\% (95\% CI, 86.7\%, 100\%) among smear positive specimens $(n=26)$ and $88.2 \%(95 \%$ CI, $63.5 \%, 98.5 \%)$ among smear negative specimens $(n=17)$.

Xpert assay, by subgroup analysis in HIV-infected patients (29 specimens), had sensitivity of $100 \%$, specificity of $100.0 \%$, PPV of $76.9 \%$, and NPV of $100 \%$ (Table 3).

\subsection{Characteristics among Culture-Confirmed Pulmonary} Tuberculosis Patients. Among 27 patients with cultureconfirmed pulmonary tuberculosis, sputum acid-fast smear was positive in 18 patients (66.7\%). Table 4 shows the
TABLE 2: Two by two contingency tables showing true positive, false positive, true negative, and false negative comparing Xpert assay (a) and sputum acid-fast smear (b) with the reference standard.

(a)

\begin{tabular}{lccc}
\hline & $\begin{array}{c}\text { Positive } \\
\text { Xpert assay }\end{array}$ & $\begin{array}{c}\text { Negative } \\
\text { Xpert assay }\end{array}$ & Total \\
\hline $\begin{array}{l}\text { Culture grew M. tuberculosis } \\
\begin{array}{l}\text { Culture grew NTM or no } \\
\text { growth }\end{array}\end{array}$ & 41 & 2 & 43 \\
\hline Total & 50 & 57 & 66 \\
\hline
\end{tabular}

NTM: nontuberculous mycobacteria, 4 in positive Xpert assay and 6 in negative Xpert assay.

(b)

\begin{tabular}{|c|c|c|c|}
\hline & $\begin{array}{l}\text { Positive sputum } \\
\text { acid-fast smear }\end{array}$ & $\begin{array}{l}\text { Negative sputum } \\
\text { acid-fast smear }\end{array}$ & Total \\
\hline $\begin{array}{l}\text { Culture grew } \\
\text { M. tuberculosis }\end{array}$ & 26 & 17 & 43 \\
\hline $\begin{array}{l}\text { Culture grew NTM } \\
\text { or no growth }\end{array}$ & 1 & 65 & 66 \\
\hline Total & 27 & 82 & 109 \\
\hline
\end{tabular}

NTM: nontuberculous mycobacteria, 10 in negative sputum acid-fast smear.

comparison of demographic and clinical characteristics between patients with positive and negative sputum acidfast smear. We found that there was no significant factor of likelihood for negative sputum acid-fast smear from both univariate and multivariate analyses.

\section{Discussion}

A recent meta-analysis that included 9,557 participants from 27 studies showed that Xpert assay of respiratory specimens had a pooled sensitivity of $89 \%(95 \%$ CI $85 \%, 92 \%)$ and specificity of $99 \%$ (95\% CI $98 \%-99 \%)$ in the diagnosis of pulmonary tuberculosis [13]. In our study that also used respiratory specimens, the overall sensitivity of Xpert assay was $95.3 \%$ and specificity was $86.4 \%$, which were slightly higher and lower than those previous studies, respectively. Compared to conventional sputum acid-fast smear that had sensitivity of $60.5 \%$ and specificity of $98.5 \%$, our study found that Xpert assay had statistically significant higher sensitivity ( $p$ value $<0.001$ ) and lower specificity ( $p$ value 0.022 ).

Data from several studies in culture-proven TB patients with acid-fast positive sputum specimens showed that Xpert assay had a pooled sensitivity of $98 \%$ (95\% CI 97\%, 99\%) in the detection of $M$. tuberculosis [12]. Our study, although with a small number of samples, also found a good concordant result with those studies; the sensitivity of Xpert assay to detect $M$. tuberculosis was $100 \%$ in acid-fast positive sputum specimens. This finding confirmed that it is unlikely for Xpert assay to miss the diagnosis of TB in patients with positive sputum acid-fast smear. However, our study showed that the sensitivity of Xpert assay for detection of M. tuberculosis in culture-proven TB specimens but negative acid-fast smear was $88.2 \%$. Our study showed considerably higher sensitivity 
TABLE 3: Sensitivity, specificity, positive predictive value, and negative predictive value of Xpert assay and sputum acid-fast smear comparing to sputum culture.

\begin{tabular}{|c|c|c|c|c|}
\hline & Xpert assay & 95\% confidence interval & Acid-fast smear & 95\% confidence interval \\
\hline \multicolumn{5}{|c|}{ Specimens from all patients $(n=109)$} \\
\hline SEN & $41 / 43=95.3 \%$ & $84.2-99.4 \%$ & $26 / 43=60.5 \%$ & $44.4-75.0 \%$ \\
\hline SPEC & $57 / 66=86.4 \%$ & $75.7-93.6 \%$ & $65 / 66=98.5 \%$ & $91.8-100 \%$ \\
\hline PPV & $41 / 50=82.0 \%$ & $68.6-91.4 \%$ & $26 / 27=96.3 \%$ & $81.0-99.9 \%$ \\
\hline NPV & $57 / 59=96.6 \%$ & $88.3-99.6 \%$ & $65 / 82=79.3 \%$ & $68.9-87.4 \%$ \\
\hline \multicolumn{5}{|c|}{ Specimens from HIV-infected patients $(n=29)$} \\
\hline SEN & $10 / 10=100 \%$ & $69.2-100 \%$ & $6 / 10=60 \%$ & $26.2-87.8 \%$ \\
\hline SPEC & $16 / 16=100 \%$ & $79.4-100 \%$ & $19 / 19=100 \%$ & $82.4-100 \%$ \\
\hline PPV & $10 / 13=76.9 \%$ & $46.2-95.0 \%$ & $6 / 6=100 \%$ & $54.1-100 \%$ \\
\hline NPV & $16 / 16=100 \%$ & $79.4-100 \%$ & $19 / 23=80 \%$ & $61.2-95.0 \%$ \\
\hline \multicolumn{5}{|c|}{ Specimens from HIV-uninfected patients $(n=77)$} \\
\hline SEN & $28 / 30=93.3 \%$ & $77.9-99.2 \%$ & $20 / 30=66.7 \%$ & $47.2-82.7 \%$ \\
\hline SPEC & $41 / 43=95.3 \%$ & $84.2-99.4 \%$ & $46 / 47=97.9 \%$ & $88.7-99.9 \%$ \\
\hline PPV & $28 / 34=82.4 \%$ & $65.5-93.2 \%$ & $20 / 21=95.2 \%$ & $76.2-99.9 \%$ \\
\hline NPV & $41 / 43=95.3 \%$ & $84.2-99.4 \%$ & $46 / 56=82.1 \%$ & $69.6-91.1 \%$ \\
\hline
\end{tabular}

SEN: sensitivity; SPEC: specificity; PPV: positive predictive value; NPV: negative predictive value.

TABLE 4: Demographic and clinical characteristics of 27 TB culture-proven patients with positive and negative sputum acid-fast smears.

\begin{tabular}{|c|c|c|c|}
\hline Characteristic & Positive acid-fast smear $(n=18)$ & Negative acid-fast smear $(n=9)$ & $p$ value \\
\hline Female & $5(27.8)$ & $6(66.7)$ & 0.097 \\
\hline Age & $48.3( \pm 17.2)$ & $61.7( \pm 22.8)$ & 0.136 \\
\hline HIV infection & $13(72.2)$ & $7(77.8)$ & 0.301 \\
\hline Presence of underlying lung disease & $5(27.8)$ & $2(22.2)$ & 0.395 \\
\hline Presence of fever & $14(77.8)$ & $4(44.4)$ & 0.083 \\
\hline Duration of illness $>7$ days & $8(44.4)$ & $4(44.4)$ & 1.000 \\
\hline Presence of chest pain & $3(16.7)$ & $2(22.2)$ & 0.726 \\
\hline Presence of dyspnea & $4(22.2)$ & $1(11.1)$ & 0.636 \\
\hline Presence of cough & $17(94.4)$ & $6(66.6)$ & 0.093 \\
\hline Hemoptysis & $1(5.6)$ & $1(11.1)$ & 1.000 \\
\hline Significant weight loss & $5(27.8)$ & $2(22.2)$ & 0.263 \\
\hline Concurrent extrapulmonary infection & $1(5.6)$ & $2(22.2)$ & 0.250 \\
\hline Abnormal CXR & $17(94.4)$ & $9(100)$ & 0.694 \\
\hline
\end{tabular}

Data are presented in number (\%), or mean \pm SD.

than those in previous studies that had a pooled sensitivity of $67 \%$ (95\% CI $60 \%, 74 \%)$ with a range from 57.1 to $76.9 \%$ [13]. Our study also showed a $100 \%$ sensitivity of Xpert assay in detecting $M$. tuberculosis in subgroup of $29 \mathrm{HIV}$ infected patients; this was higher than $84 \%$ and $69.6 \%$ in previous studies $[14,15]$. The difference in sensitivity of Xpert assay on detection of $M$. tuberculosis among studies may be resulted from the difference in inclusion criteria. Inclusion of patients with minimal symptoms or normal chest X-ray that may have low bacillary load could result in higher rate of negative results from Xpert assay. In addition, the technique to obtain sputum specimens varied among studies. Sputum induction may cause less bacillary load compared to the expectorated sputum $[12,16]$.
Although WHO endorsed the wide use of Xpert MTB/RIF assay, this test is expensive and available only in large medical centers in Thailand. Using this assay in all patients with clinically suspected tuberculosis may neither be possible nor cost-effective in resource-limited settings in particular where the laboratory technicians have high experience in microscopy. As mentioned above, our study and other studies all confirm that positive acid-fast smear correlates well with Xpert assay and TB culture; patients with positive acid-fast smear may not have benefit from Xpert assay in detecting $\mathrm{TB}$ but may have benefit in detecting rifampin resistance if it exists. In order to identify predicting factors for having negative sputum acid-fast smear, we compared demographic and clinical characteristics among 
patients with culture-confirmed pulmonary tuberculosis who had positive and negative sputum acid-fast smears. Patient who has one of these factors, if any, and has negative sputum acid-fast smear may have benefit from Xpert assay. Unfortunately, we could not identify any factors associated with negative sputum acid-fast smear in patients with tuberculosis. Failure to demonstrate that may be due to small number of patients in this study and further studies with larger sample size may be warranted.

Our study demonstrated the overall specificity of $86.4 \%$ (75.7-93.6\%), which was slightly lower than those in previous studies that had a range from $94 \%$ to $100 \%$ [12]. Five sputum specimens from 4 patients in our study had positive Xpert assay but negative TB culture and negative acid-fast smear. One patient with HIV infection had cervical lymphadenopathy and $M$. tuberculosis was isolated from his lymph node culture. Two patients had other sputum specimens that were acid-fast positive but cultures were not performed. This suggested that MGIT culture may not be a good reference standard. Using the reference standard with low sensitivity could lead to a lower specificity of the test. In fact, Xpert assay may be more appropriate for reference standard since previous analytical study demonstrated that MGIT culture can detect $M$. tuberculosis at the level of $5 \times 10^{5} \mathrm{cfu} / \mathrm{mL}$ [17] while the Xpert assay can detect M. tuberculosis at the level of as low as $131 \mathrm{cfu} / \mathrm{mL}$ or 5 genome copies of DNA $[6,18]$.

Interestingly, 10 samples from 6 patients in our study grew NTM. Four samples from 3 patients were weakly positive by Xpert assay. In one patient who produced 2 sputum specimens, one specimen grew $M$. tuberculosis and the other grew NTM. We, therefore, believe that the positive result in these 4 specimens may be from infection of both NTM and $M$. tuberculosis in the same patients. Data from previous studies also confirm that it is not common for Xpert assay to produce a false positive result in NTM growth specimens [13]. Recent analytical study also found that Xpert assay can correctly detect $M$. tuberculosis in patients who had mixed infection with high number of NTM and low number of $M$. tuberculosis [19].

Of note, invalid and error results from Xpert assay occurred in 4 of 127 specimens (3.1\%) whereas contaminated culture occurred in 14 of 127 specimens (11.0\%). In addition, median turnaround time from sputum collection to get Xpert results was faster than from culture results. Due to the timetaking process to prepare and run the test for each specimen, we decide to run the test once we have four samples ready instead of one at a time. Therefore, the median turnaround time for Xpert assay in our study was 6 days, comparing to 45 days for culture results. These demonstrated that Xpert assay was more reliable than sputum culture for M. tuberculosis and had more rapid turnaround time.

Our study has several limitations. First, there was a high rate of contaminated culture specimens (11.0\%). This may indicated poor technique of specimen collection and decontamination. Second, the relatively small numbers of participants limited the study power. Finally, since there were only 3 specimens from one patient with rifampin resistance in this study, data was not sufficient to evaluate Xpert performance in detecting rifampin resistance.

\section{Conclusions}

This study demonstrated good sensitivity and specificity of Xpert assay in detecting $M$. tuberculosis from respiratory specimens. This is particularly helpful in making a rapid diagnosis and initiating prompt treatment of tuberculosis in patients who had negative sputum acid-fast smear.

\section{Conflict of Interests}

All authors declared no conflict of interests.

\section{Acknowledgments}

This study was supported by the National Research University Project under Thailand's Office of the Higher Education Commission. The funders had no role in study design, data collection and analysis, decision to publish, or preparation of the paper.

\section{References}

[1] World Health Organization (WHO), Global Tuberculosis Report 2013, WHO, Geneva, Switzerland, 2013, http://apps.who.int/ iris/bitstream/10665/91355/1/9789241564656_eng.pdf.

[2] M. J. Reid and N. S. Shah, "Approaches to tuberculosis screening and diagnosis in people with HIV in resource-limited settings," The Lancet Infectious Diseases, vol. 9, no. 3, pp. 173-184, 2009.

[3] H. Getahun, M. Harrington, R. O'Brien, and P. Nunn, "Diagnosis of smear-negative pulmonary tuberculosis in people with HIV infection or AIDS in resource-constrained settings: informing urgent policy changes," The Lancet, vol. 369, no. 9578, pp. 2042-2049, 2007.

[4] S. C. van Kampen, R. M. Anthony, and P. R. Klatser, "The realistic performance achievable with mycobacterial automated culture systems in high and low prevalence settings," BMC Infectious Diseases, vol. 10, article 93, 2010.

[5] K. Siddiqi, M.-L. Lambert, and J. Walley, "Clinical diagnosis of smear-negative pulmonary tuberculosis in low-income countries: the current evidence," Lancet Infectious Diseases, vol. 3, no. 5, pp. 288-296, 2003.

[6] R. Blakemore, E. Story, D. Helb et al., "Evaluation of the analytical performance of the Xpert MTB/RIF assay," Journal of Clinical Microbiology, vol. 48, no. 7, pp. 2495-2501, 2010.

[7] K. Chang, W. Lu, J. Wang et al., "Rapid and effective diagnosis of tuberculosis and rifampicin resistance with Xpert MTB/RIF assay: a meta-analysis," Journal of Infection, vol. 64, no. 6, pp. 580-588, 2012.

[8] C. C. Boehme, P. Nabeta, D. Hillemann et al., "Rapid molecular detection of tuberculosis and rifampin resistance," The New England Journal of Medicine, vol. 363, no. 11, pp. 1005-1015, 2010.

[9] D. Hillemann, S. Rüsch-Gerdes, C. Boehme, and E. Richter, "Rapid molecular detection of extrapulmonary tuberculosis by the automated genexpert MTB/RIF system," Journal of Clinical Microbiology, vol. 49, no. 4, pp. 1202-1205, 2011.

[10] B. Malbruny, G. Le Marrec, K. Courageux, R. Leclercq, and V. Cattoir, "Rapid and efficient detection of Mycobacterium 
tuberculosis in respiratory and non-respiratory samples," The International Journal of Tuberculosis and Lung Disease, vol. 15, no. 4, pp. 553-555, 2011.

[11] A. Vassall, S. van Kampen, H. Sohn et al., "Rapid diagnosis of tuberculosis with the Xpert MTB/RIF assay in high burden countries: a cost-effectiveness analysis," PLoS Medicine, vol. 8, no. 11, Article ID e1001120, 2011.

[12] Cepheid, Xpert MTB/RIF Assay (Package Insert), Cepheid, Sunnyvale, Calif, USA, 2013.

[13] K. R. Steingart, I. Schiller, D. J. Horne, M. Pai, C. C. Boehme, and N. Dendukuri, "Xpert MTB/RIF assay for pulmonary tuberculosis and rifampicin resistance in adults," The Cochrane Database of Systematic Reviews, vol. 1, Article ID CD009593, 2013.

[14] L. E. Scott, K. McCarthy, N. Gous et al., "Comparison of Xpert MTB/RIF with other nucleic acid technologies for diagnosing pulmonary tuberculosis in a high HIV prevalence setting: a prospective study," PLoS Medicine, vol. 8, no. 7, Article ID e1001061, 2011.

[15] G. Theron, J. Peter, R. van Zyl-Smit et al., "Evaluation of the Xpert MTB/RIF assay for the diagnosis of pulmonary tuberculosis in a high HIV prevalence setting," American Journal of Respiratory and Critical Care Medicine, vol. 184, no. 1, pp. 132140, 2011.

[16] H. Sohn, A. D. Aero, D. Menzies et al., "Xpert MTB/RIF testing in a low tuberculosis incidence, high-resource setting: limitations in accuracy and clinical impact," Clinical Infectious Diseases, vol. 58, no. 7, pp. 970-976, 2014.

[17] M.-C. Yu, H.-Y. Chen, M.-H. Wu et al., "Evaluation of the rapid MGIT TBc identification test for culture confirmation of Mycobacterium tuberculosis complex strain detection," Journal of Clinical Microbiology, vol. 49, no. 3, pp. 802-807, 2011.

[18] Switzerland World Health Organization, Rapid Implementation of the Xpert MTB/RIF Diagnostic Test: Technical and Operational "How-To"; Practical Considerations, Switzerland World Health Organization, Geneva, Switzerland, 2011, http://whqlibdoc.who .int/publications/2011/9789241501569_eng.pdf.

[19] D. Helb, M. Jones, E. Story et al., "Rapid detection of Mycobacterium tuberculosis and rifampin resistance by use of ondemand, near-patient technology," Journal of Clinical Microbiology, vol. 48, no. 1, pp. 229-237, 2010. 


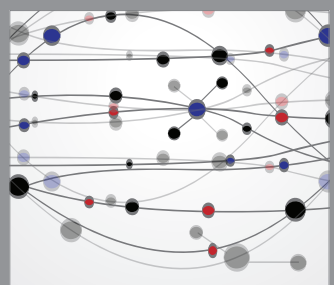

The Scientific World Journal
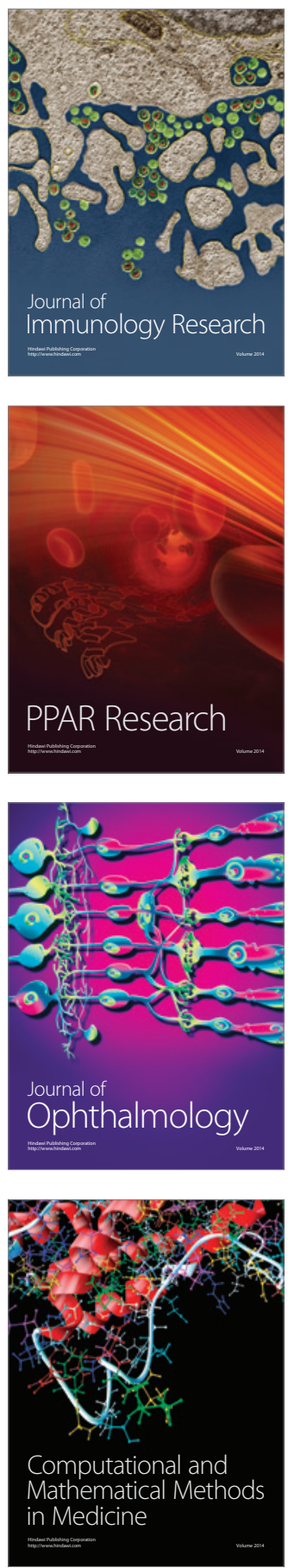

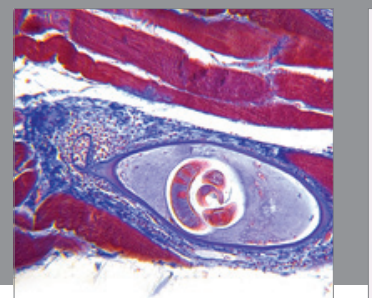

Gastroenterology

Research and Practice
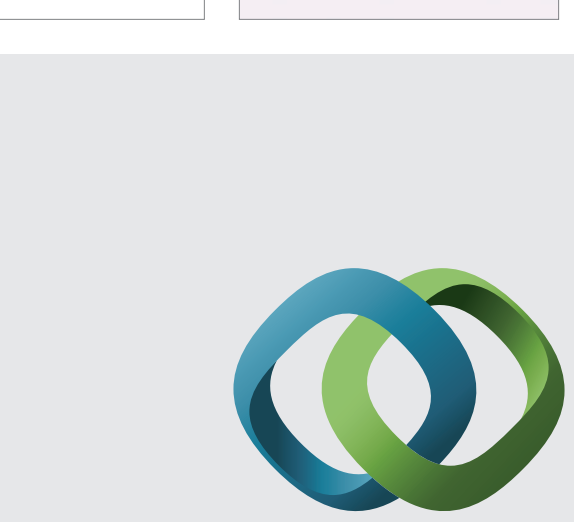

\section{Hindawi}

Submit your manuscripts at

http://www.hindawi.com
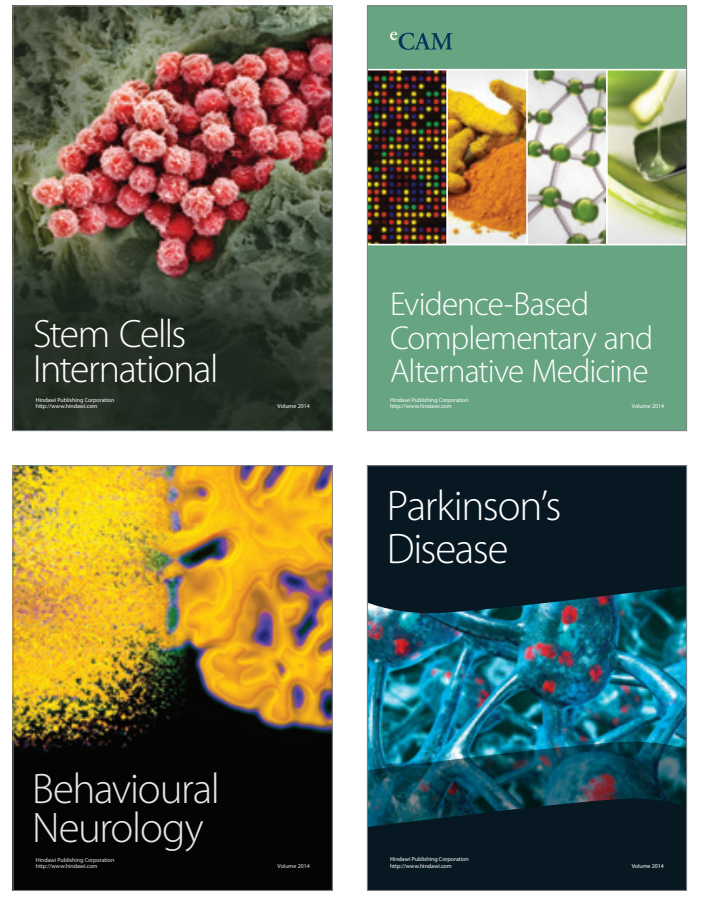
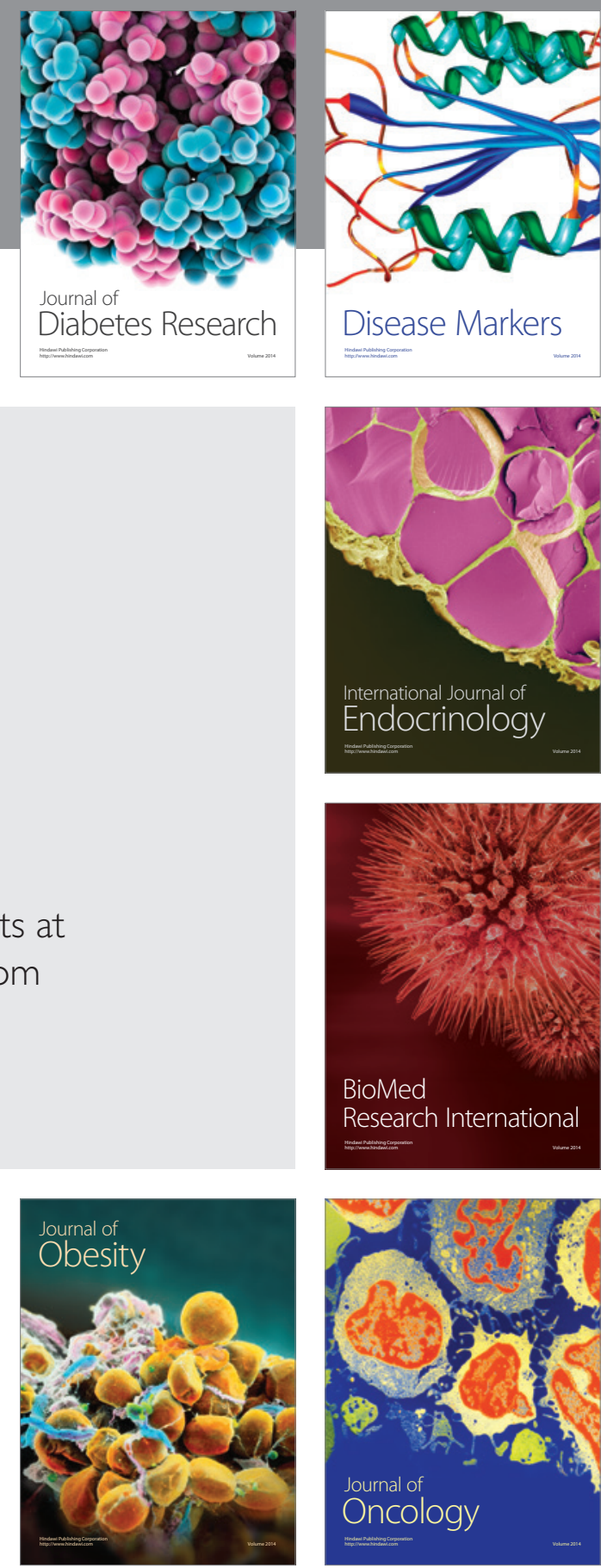

Disease Markers
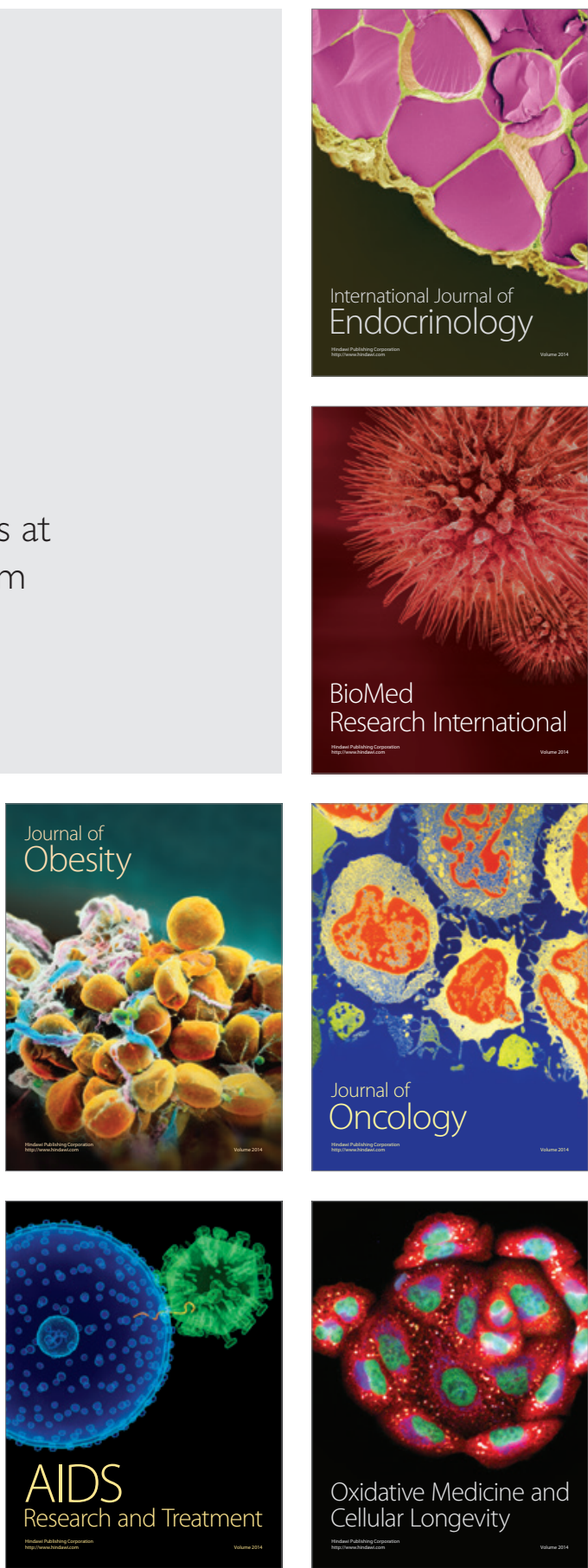